\title{
P-Band Induced Self-Organization and Dynamics with Repulsively Driven Ultracold Atoms in an Optical Cavity
}

\section{Journal Article}

\section{Author(s):}

Zupancic, Philip; Dreon, Davide (D); Li, Xiangliang; Baumgärtner, Alexander; Morales, Andrea; Zheng, Wei; Cooper, Nigel R.; Esslinger, Tilman; Donner, Tobias Ulrik (D)

\section{Publication date:}

2019-12-06

\section{Permanent link:}

https://doi.org/10.3929/ethz-b-000385907

\section{Rights / license:}

In Copyright - Non-Commercial Use Permitted

Originally published in:

Physical Review Letters 123(23), https://doi.org/10.1103/physrevlett.123.233601

\section{Funding acknowledgement:}

182650 - Interplay between Topology, Interactions and Dissipation in Driven Quantum Many-Body Systems (SNF)

175329 - Non-equilibrium dynamics in Atomic systems for QUAntum Simulation (SNF)

742579 - Mass, heat and spin transport in interlinked quantum gases (EC)

721465 - Collective effects and optomechanics in ultra-cold matter (EC) 


\title{
$P$-Band Induced Self-Organization and Dynamics with Repulsively Driven Ultracold Atoms in an Optical Cavity
}

\author{
P. Zupancic $\odot,{ }^{1}$ D. Dreon $\odot,{ }^{1}$ X. Li, ${ }^{1}$ A. Baumgärtner, ${ }^{1}$ A. Morales $\odot,{ }^{1}$ \\ W. Zheng, ${ }^{2}$ N. R. Cooper, ${ }^{2}$ T. Esslinger, ${ }^{1}$ and T. Donner $\oplus^{1, *}$ \\ ${ }^{1}$ Institute for Quantum Electronics, Eidgenössische Technische Hochschule Zürich, Otto-Stern-Weg 1, 8093 Zurich, Switzerland \\ ${ }^{2}$ T.C.M. Group, Cavendish Laboratory, University of Cambridge, JJ Thomson Avenue, Cambridge CB3 OHE, United Kingdom
}

(Received 27 May 2019; published 2 December 2019)

\begin{abstract}
We investigate a Bose-Einstein condensate strongly coupled to an optical cavity via a repulsive optical lattice. We detect a stable self-ordered phase in this regime, and show that the atoms order through an antisymmetric coupling to the $P$ band of the lattice, limiting the extent of the phase and changing the geometry of the emergent density modulation. Furthermore, we find a nonequilibrium phase with repeated intense bursts of the intracavity photon number, indicating nontrivial driven-dissipative dynamics.
\end{abstract}

DOI: 10.1103/PhysRevLett.123.233601

Strong coupling between ultracold matter and quantized light fields can be achieved by placing a quantum gas in a high-finesse optical cavity. Their interplay generates nonlinear atom-field dynamics that forms the basis for exploring collective many-body phenomena at the interface between quantum optics and condensed matter physics [1-11]. A central phenomenon in this approach is the self-organization of a Bose-Einstein condensate (BEC) in a cavity mode [12], when the atoms are illuminated from the side by a reddetuned, attractive standing wave pump lattice. Above a critical lattice depth, the particles are dragged into the intensity maxima of an emerging interference potential, thereby maximizing the scattering from the pump lattice to the cavity mode. The self-organization process is a second order phase transition at which the atoms reduce their potential energy in the modified potential landscape by a larger amount than the kinetic energy cost of the additional crystalline structure. This self-consistent ordering of atoms and light has become an experimental model system for driven-dissipative quantum phases [12-21].

Theoretical studies have made predictions for possible self-ordered phases in a parameter regime where a quantum gas is coupled to a cavity via a blue-detuned optical lattice, which repels atoms from the intensity maxima [22-25]. Naively one could expect that self-organization is prohibited, since the buildup of any additional repulsive potential seems to cost energy. However, since the light field scattered by the atoms into the cavity is out of phase from the pump lattice field, destructive interference occurs at the position of the atoms. This carves out parts of the repulsive pump lattice potential, lowers the potential energy, and makes self-organization also possible for a blue-detuned pump (see Fig. 1). In such a configuration, limit cycles and chaos were recently predicted $[22,24,26,27]$ and connected to dynamic phenomena such as time crystals [28].
Experiments, however, have so far been limited to the case of red-detuned, attractive pump fields. In this Letter we report on self-organization with a repulsive optical lattice and identify the parameter regime for a stable phase, both experimentally and theoretically. We further discover

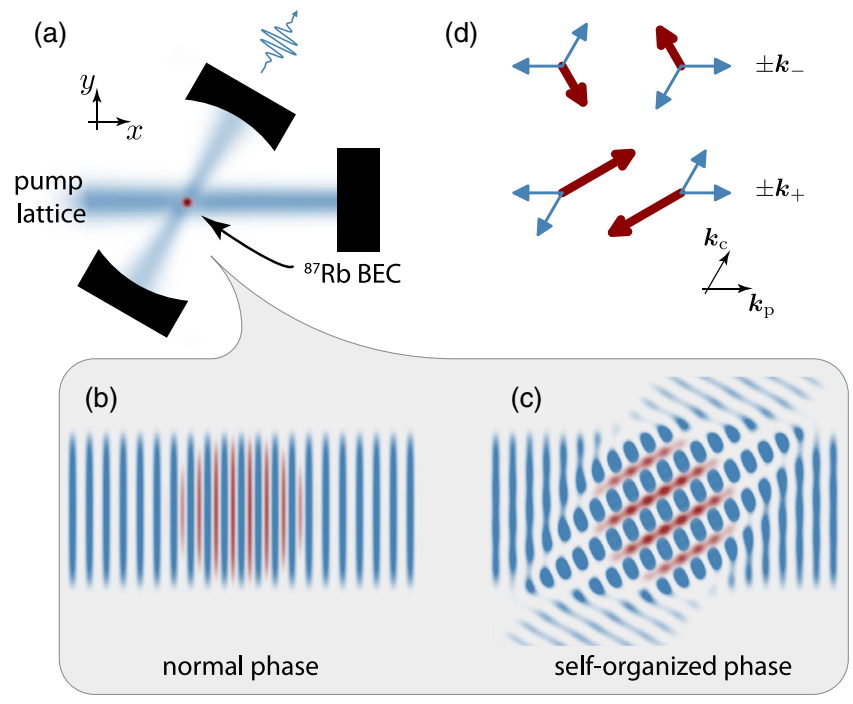

FIG. 1. (a),(b) We prepare a BEC of ${ }^{87} \mathrm{Rb}$ atoms in the initially unpopulated mode of an optical resonator, and expose it to a repulsive optical lattice (blue) localizing the atoms (red) in the field minima. (c) Populating the cavity mode with photons can become energetically favorable for the system because interference between the pump and the cavity fields gives the atoms more space to expand close to the nodes of the total field. The atoms form a Bragg grating that allows scattering into the cavity mode. Photons leaking from the cavity are detected. (d) The atoms recoil and acquire momentum (red arrows) from scattering photons (blue arrows). These momenta are $\pm \boldsymbol{k}_{-}= \pm\left(\boldsymbol{k}_{c}-\boldsymbol{k}_{p}\right)$ (upper processes) and $\pm \boldsymbol{k}_{+}= \pm\left(\boldsymbol{k}_{c}+\boldsymbol{k}_{p}\right)$ (lower processes). 
experimentally a dynamic region, where the intracavity light field shows repeated pulses.

Our experimental setup is depicted in Fig. 1(a). The experiments start with the creation of a BEC of $N=$ $2.7(1) \times 10^{5}{ }^{87} \mathrm{Rb}$ atoms by optical evaporation in a crossed dipole trap [29]. The BEC is placed in the mode of an optical resonator with a decay rate of $\kappa=2 \pi \times 147(4) \mathrm{kHz}$ and atom-cavity coupling $g_{0}=2 \pi \times 1.95(10) \mathrm{MHz}$. We apply the pump lattice beam to the atoms at an angle of $60(1)^{\circ}$ with respect to the cavity mode. The lattice depth $V_{p}$ is ramped up linearly over time in $50 \mathrm{~ms}$ from 0 to $17(1)$ $E_{\text {rec }}$, where $E_{\text {rec }}=2 \pi \hbar \times 3.77 \mathrm{kHz}$ and $\hbar$ is the reduced Planck's constant. The pump beam has a wave vector $\boldsymbol{k}_{p}=$ $(2 \pi / \lambda) \boldsymbol{x}$ with wavelength $\lambda=780.1 \mathrm{~nm}$, and corresponding frequency $\omega_{p}$. It is blue detuned by $\Delta_{a}=\omega_{p}-\omega_{a}=$ $2 \pi \times 76.6(1) \mathrm{GHz}$ with respect to the $D 2$ line of ${ }^{87} \mathrm{Rb}$ at $\omega_{a}$ (see [29] for the effect of changing $\Delta_{a}$ ), and detuned by $\Delta_{c}=\omega_{p}-\omega_{c}$ with respect to the resonance $\omega_{c}$ of the cavity mode with wave vector $\boldsymbol{k}_{c}$. We detect photons leaking from the cavity with a single-photon counting module and convert the detection rate to an intracavity lattice depth. We use this cavity lattice depth as the order parameter of the phase transition.

Repeating this experiment for different cavity detunings $\Delta_{c}$, we construct the phase diagram of the system as shown in Fig. 2(a). Blue regions indicate finite mean intracavity photon numbers which we identify with a self-ordered phase. For small lattice depths $V_{p}$ and in the vicinity of $\Delta_{c}=0$, the phase boundary is approximately linear. Its slope changes for increasing lattice depth, forming a tip of the self-organized phase at a finite cavity detuning $\Delta_{c} \approx-2 \pi \times 3 \mathrm{MHz}$, from where the phase boundary bends up again and converges towards $\Delta_{c}=0$ for large pump lattice depths. This is in stark contrast to the case of a reddetuned pump lattice $\left(\Delta_{a}<0\right)$, where the phase boundary is monotonic such that a phase transition to the self-ordered phase exists for any $\Delta_{c}<0$ [12]. For finite positive $\Delta_{c}$, we observe a self-ordered phase which however also disappears towards higher pump lattice depths, followed by a few lines in the phase diagram. In the red-detuned case, no self-organization is observed for positive $\Delta_{c}$ due to the opposite sign of the dispersive shift.

Absorption images after ballistic expansion of the atoms reveal their momentum distribution. In the normal phase, the atoms are localized in the nodes of the pump lattice [Fig. 2(b)]. In the self-ordered phase, the atoms acquire a strong density modulation in the direction $\boldsymbol{k}_{-}=\boldsymbol{k}_{c}-\boldsymbol{k}_{p}$ [Fig. 2(c)]. This modulation has twice the pump lattice periodicity $(\lambda)$ due to the chosen angle of $60^{\circ}$ between pump and cavity. The qualitative experimental results, however, do not depend on this choice. In real space, this corresponds to a Bragg grating scattering photons from the pump into the cavity. While four different scattering processes are possible as depicted in Fig. 1(d), mostly the scattering processes populating the momenta at $\pm \boldsymbol{k}_{-}$occur

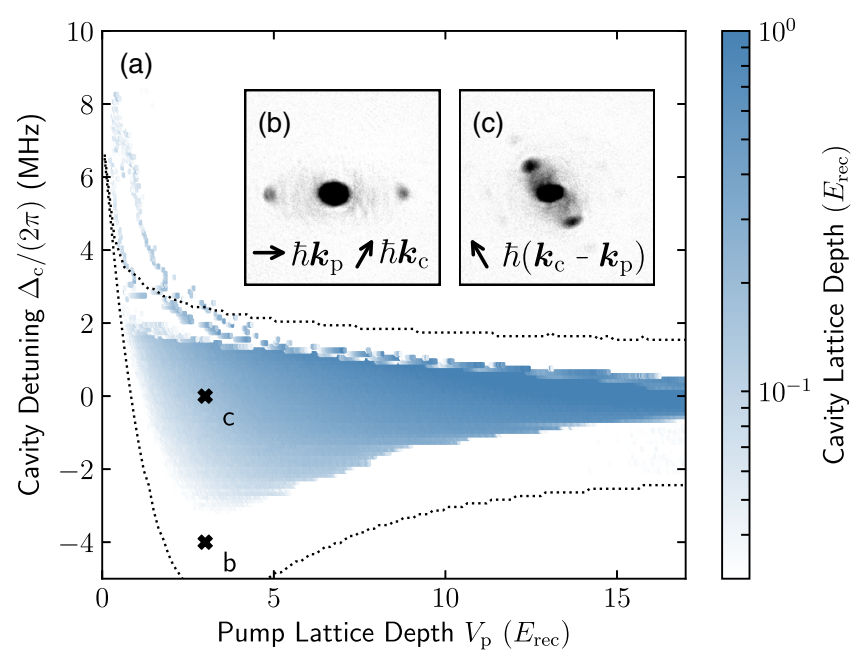

FIG. 2. Self-organized phase with a repulsive pump. (a) Phase diagram showing the intracavity lattice depth as a function of pump lattice depth and cavity detuning. A self-ordered phase with limited extent, identified by a finite lattice in the cavity, can be identified. The dotted lines are the phase boundaries obtained from numerical mean-field calculations. The deviation from the data can be attributed to finite temperatures [29]. Insets display atomic momentum distributions for a pump lattice of 3.0(2) $E_{\mathrm{rec}}$ recorded with absorption imaging after ballistic expansion. (b) For $\Delta_{c}=-2 \pi \times 4.0(1) \mathrm{MHz}$, the system is in the normal phase with momentum components at 0 and $\pm 2 \hbar \boldsymbol{k}_{p}$. In real space, this distribution corresponds to the density modulation of the pump lattice with $\lambda / 2$ spacing. (c) At $\Delta_{c}=0$, the system is self-organized, and the atoms assume a 1D density modulation with $\lambda$ spacing with momentum components $\pm \hbar\left(\boldsymbol{k}_{c}-\boldsymbol{k}_{p}\right)$. Both the pump lattice modulation at $\pm 2 \hbar \boldsymbol{k}_{p}$ and the recoil from the other scattering process at $\pm \hbar\left(\boldsymbol{k}_{c}+\boldsymbol{k}_{p}\right)$ [see Fig. 1(d)] are suppressed.

[upper graphs in Fig. 1(d)], and the processes populating the momenta at $\pm \boldsymbol{k}_{+}= \pm\left(\boldsymbol{k}_{c}+\boldsymbol{k}_{p}\right)$ are suppressed. In the selfordered phase also the density modulation by the pump lattice is suppressed because of the destructive interference reducing the pump potential, as can be seen by the decreased population of the $\pm 2 \hbar \boldsymbol{k}_{p}$ momenta in Fig. 2(c) compared to Fig. 2(b). Again, this is in contrast to the case of a reddetuned pump lattice, where all possible momentum states are macroscopically populated.

These observations can be understood from analyzing the Hamiltonian of the atom-cavity system consisting of the following five terms [30]:

$$
\hat{\mathcal{H}}=\hat{\mathcal{H}}_{\text {ph }}+\hat{\mathcal{H}}_{\text {kin }}+\hat{\mathcal{H}}_{\text {pot }}^{\text {pump }}+\hat{\mathcal{H}}_{\text {pot }}^{\text {cavity }}+\hat{\mathcal{H}}_{\text {pot }}^{\text {coupl }}
$$

The atoms self-order when the scattering of photons lowers the energy of the system, i.e., when the gain in potential energy exceeds the energy cost of cavity photons, $\hat{\mathcal{H}}_{\mathrm{ph}}=-\hbar \Delta_{c} \hat{a}^{\dagger} \hat{a}$, plus the kinetic energy cost caused by recoiling atoms, $\hat{\mathcal{H}}_{\text {kin }}=\left(\hat{p}^{2} / 2 m\right)$. Here, $\Delta_{c}$ is the detuning 

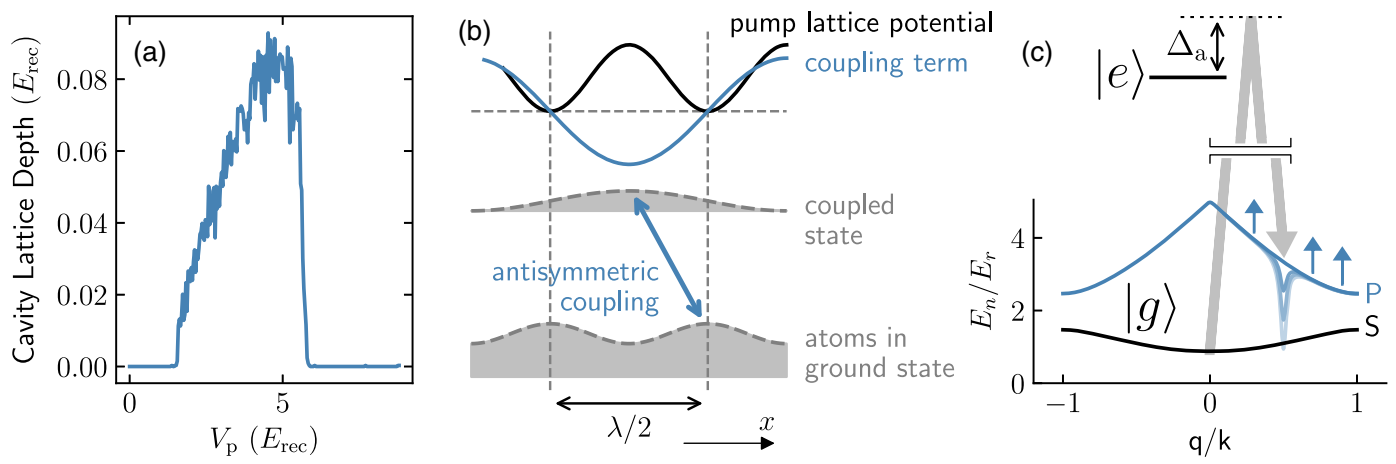

FIG. 3. Finite extent of the self-organized phase. (a) Cut through the phase diagram for $\Delta_{c}=-2 \pi \times 2$ MHz showing the cavity lattice depth rising from and returning to 0 . (b) The atoms (gray) get localized to the nodes of the repulsive standing wave field (black) along the $x$ direction. Taking the atomic density maxima as origin of the coordinate system, the coupling term with the double period (blue) turns into a sine. It thus couples to a state of opposite symmetry, as indicated. (c) This opposite-symmetry state lives in the $P$ band of the pump lattice. With increasing pump lattice depth, the coupling term leads to a mode softening at wave vector $\boldsymbol{k}_{-}=\boldsymbol{k}_{c}-\boldsymbol{k}_{p}$ (pale blue lines), and finally to self-organization. At the same time, the increasingly deep pump lattice increases the energy of the $P$ band (blue arrows). For deeper lattices, this effect becomes dominant and leads to the end of the self-ordered phase.

of the pump frequency relative to the cavity resonance, $\hat{a}^{\dagger}$ and $\hat{a}$ create and annihilate cavity photons, respectively, $\hat{p}$ is the atomic momentum operator, and $m$ the atomic mass. The potential energy has the three terms

$$
\begin{aligned}
& \hat{\mathcal{H}}_{\text {pot }}^{\text {pump }}=V_{p} \cos ^{2}\left(\boldsymbol{k}_{p} \cdot \hat{\boldsymbol{r}}\right), \\
& \hat{\mathcal{H}}_{\text {pot }}^{\text {cavity }}=U_{0} \hat{a}^{\dagger} \hat{a} \cos ^{2}\left(\boldsymbol{k}_{c} \cdot \hat{\boldsymbol{r}}\right), \\
& \hat{\mathcal{H}}_{\mathrm{pot}}^{\text {coupl }}=\sqrt{V_{p} U_{0}}\left(\hat{a}+\hat{a}^{\dagger}\right) \cos \left(\boldsymbol{k}_{c} \cdot \hat{\boldsymbol{r}}\right) \cos \left(\boldsymbol{k}_{p} \cdot \hat{\boldsymbol{r}}\right),
\end{aligned}
$$

where $U_{0}=0.012 E_{\text {rec }}$ is the dispersive cavity shift per atom.

Equation (4) describes the coupling of the pump lattice to the light field of the cavity and the atomic density. This leads to a polariton mode, a coupled excitation of the cavity light field and the atomic density at momenta $\pm \boldsymbol{k}_{ \pm}$. With increasing pump lattice depth, its energy is reduced [31]. The polariton mode softens and approaches zero energy at the critical point, manifesting a continuous phase transition.

Although Eq. (4) couples equally to the $\boldsymbol{k}_{-}$and $\boldsymbol{k}_{+}$modes, there is a strong imbalance between their populations in the self-ordered phase [Fig. 2(c)] for two reasons. The first is the difference in their respective kinetic energies of $1 E_{\text {rec }}$ and $3 E_{\text {rec }}$, respectively. The second, more intricate one is that Eqs. (2) and (3) have finite matrix elements coupling the two modes with $\left\langle\boldsymbol{k}_{-}\left|\left(\hat{\mathcal{H}}_{\text {pot }}^{\text {pump }}+\hat{\mathcal{H}}_{\text {pot }}^{\text {cavity }}\right)\right| \boldsymbol{k}_{+}\right\rangle \propto 1 / \Delta_{a}$. For $\Delta_{a}>0$, a population of both modes increases the total energy of the system and is thus disfavored, opposite to the case of $\Delta_{a}<0$.

The finite extent of the self-ordered phase for large pump lattice depths and negative cavity detunings [Figs. 2(a) and 3(a)] can be explained with a symmetry argument [see Fig. 3(b)]: For deep lattices, the atoms in the ground state localize in the minima of the potential in Eq. (2) $\propto \cos ^{2}\left(\boldsymbol{k}_{p} \cdot \boldsymbol{r}\right)$, which for $\Delta_{a}>0$ are located at the nodes of the light field. The maxima of the atomic density distribution are thus shifted by $\lambda / 4$ with respect to the pump lattice. Accordingly, taking the atomic density maxima as the origin of the coordinate system, the coupling term in Eq. (4) becomes $\propto \sin \left(\boldsymbol{k}_{p} \cdot \boldsymbol{r}\right)$. Being an odd operator, it couples the atoms in the ground state to a state of opposite parity. Because of its opposite symmetry, this state is in the $P$ band of the pump lattice and localized at the maxima of the pump potential. As we ramp up the pump lattice, the coupling that leads to the mode softening is enhanced [pale blue lines in Fig. 3(c)], enabling self-organization. But with further increasing pump lattice depth, the band gap increases (blue arrows), which pushes the system out of the self-ordered phase again. The phase boundaries arise from the competition between band gap and mode softening.

The above arguments are confirmed by going to the mean-field limit of Eq. (1) and solving the steady-state equations in perturbation theory [29]. We find the criterion for superradiance for $N$ atoms

$$
1<\frac{4 N U_{0} \tilde{\Delta}_{c}}{\tilde{\Delta}_{c}^{2}+\kappa^{2}} V_{p} \chi_{\mathrm{sp}}\left(V_{p}\right)
$$

where we defined the dispersively shifted cavity detuning $\tilde{\Delta}_{c}=\Delta_{c}-N U_{0} / 2$ and the single-particle response function

$$
\chi_{\mathrm{sp}}\left(V_{p}\right)=\sum_{n, \boldsymbol{k}} \frac{\left|\left\langle\psi_{n}^{(0)}(\boldsymbol{k})|\hat{\Theta}| \psi_{1}^{(0)}(\mathbf{0})\right\rangle\right|^{2}}{E_{1}^{(0)}(\mathbf{0})-E_{n}^{(0)}(\boldsymbol{k})} .
$$

$\psi_{n}^{(0)}(\boldsymbol{k})$ are the unperturbed Bloch wave functions in the $n$ th band of the pump lattice with eigenenergies $E_{n}^{(0)}$, and $\hat{\Theta}=\cos \left(\boldsymbol{k}_{p} \cdot \hat{\boldsymbol{r}}\right) \cos \left(\boldsymbol{k}_{c} \cdot \hat{\boldsymbol{r}}\right)$. 
Taking into account only the two lowest bands and taking the limit of weak lattices we can evaluate [29]

$$
-V_{p} \chi_{\mathrm{sp}}\left(V_{p}\right) \approx \frac{V_{p}}{3 E_{\mathrm{rec}}}\left(1-\frac{V_{p}}{8 E_{\mathrm{rec}}}\right) .
$$

This function first rises to a maximum value at $V_{p}=4 E_{\text {rec }}$, which can be enough to enter the self-organized phase depending on $\Delta_{c}$, but then decreases leading to the end of the self-ordered phase. This reproduces the form of the lower edge of the ordered phase in Fig. 2(a). This is very different from the familiar self-organization for $\Delta_{a}<0$, for which the pump lattice-coupling the BEC to the lowest band-reduces the bandwidth $E_{1}^{(0)}(\boldsymbol{k})-E_{1}^{(0)}(\mathbf{0})$ and increases the overlap $\left|\left\langle\psi_{1}^{(0)}(\boldsymbol{k})|\hat{\Theta}| \psi_{1}^{(0)}(\mathbf{0})\right\rangle\right|^{2}$ and thus works in favor of, rather than against, self-organization. The repulsive nature of the potentials and the band effects counteracting the mode softening lead to a marginal energy difference between the normal and the self-ordered phase. This energy gain of the ordered phase goes down with detuning so that finite temperatures shift the lower tip in the phase diagram up [cf. theory lines in Fig. 2(a) and see [29] for data].

Qualitatively different behavior arises for $\Delta_{c}>0$ in Fig. 2(a), where we find striking dynamical effects. With increasing pump strength the system enters the self-ordered phase, but then the cavity photon number spikes and the order parameter vanishes. This process reoccurs several times. To disentangle pump from time dynamics, we perform quench experiments in which we ramp up the pump lattice depth at fixed $\Delta_{c}=2 \pi \times-10 \mathrm{MHz}$ in the nonorganized phase. We then jump the detuning to the parameter region of interest, and subsequently, keeping pump and detuning constant, record the time evolution of the cavity field. Figure 4(a) shows an exemplary trace outside the stable self-ordered phase at $\Delta_{c}=2 \pi \times 2.0(1) \mathrm{MHz}$ and $V_{p}=4.1(2) E_{\text {rec }}$. We observe repeating pulses with a duration of 3(1) $\mu$ s and a cavity lattice depth of 32(17) $E_{\text {rec }}$, much deeper than the pump lattice, appearing after an initial buildup time. Taking absorption images of the atomic momentum distribution within a millisecond of a spike reveals the population of very high momentum states [Figs. 4(b) and 4(c)], as expected for deep lattices. The timescale of buildup and decay of the cavity field spikes is consistent with our cavity decay rate $\kappa=2 \pi \times 147(4) \mathrm{kHz}$. We find the same behavior everywhere between the boundary of the steady-state phase and $\Delta_{c}=N U_{0} / 2$, leading to the schematic phase diagram of Fig. 4(d).

A basic understanding for the appearance of dynamic features for $0<\Delta_{c}<N U_{0} / 2$ can be gained by neglecting dissipation for the moment and considering the free energy landscape of the system. Figure 4(e) shows the numerically obtained free energy as a function of the cavity field $\langle\hat{a}\rangle$ for $\Delta_{c}=2 \pi \times 2 \mathrm{MHz}$ and a precritical pump power. The term $-\Delta_{c} \hat{a}^{\dagger} \hat{a}$ in Eq. (1) indicates that the system can lower its

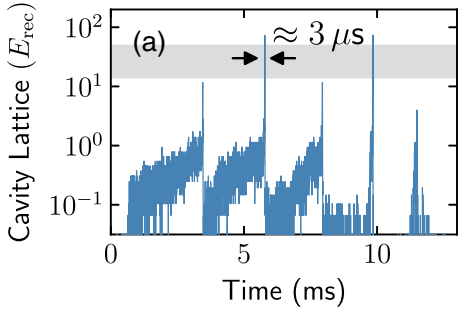

(d)
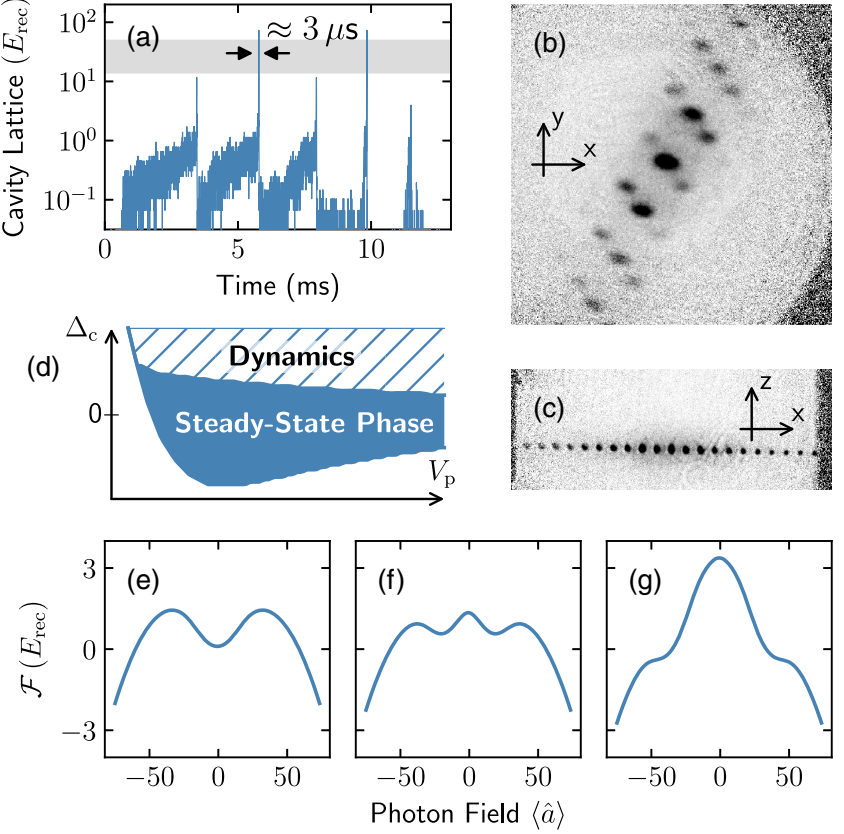

FIG. 4. Superradiant dynamics. (a) Time trace of the cavity lattice depth after a far-detuned ramp-up of the pump to $4.1 E_{\mathrm{rec}}$ and a subsequent jump of detuning from $\Delta_{c}=2 \pi \times-10 \mathrm{MHz}$ to $\Delta_{c}=2 \pi \times 2 \mathrm{MHz}$ at time 0 . The difference in spike heights stems from the finite photon count rate at which the single photon counting module saturates [29]. The gray bar indicates the estimated uncertainty. (b),(c) Momentum distribution from absorption imaging after ballistic expansion after a photon spike in the cavity-pump-plane $[x y,(b)]$ and an orthogonal plane $[x z,(c)]$. Very high momenta up to $\pm 20 \hbar \boldsymbol{k}_{c}$ are visible, possibly limited by the aperture of the imaging system. (d) Schematic phase diagram with stable and dynamic regions. (e)-(g) Numerical calculations of the free energy per atom as a function of the cavity photon field for $\Delta_{c}=2 \pi \times 2 \mathrm{MHz}$ and pump lattice depths of $0.2 E_{\mathrm{rec}}$ (normal phase with local minimum at $\langle\hat{a}\rangle=0$ ), $3.4 E_{\text {rec }}$ (self-ordered phase with local minima at a finite photon number) and $13 E_{\mathrm{rec}}$ (spike region, no local minimum).

energy in the rotating frame with more photons, but the local minimum at $\langle\hat{a}\rangle=0$ keeps the system in the normal phase. With increasing pump power, local minima at finite cavity fields develop and the atoms self-organize [Fig. 4(f)]. Uniquely for $\Delta_{a}>0$ [29] and in contrast to the case $\Delta_{c}<0$, here the self-organized state is metastable. In fact, deeper in the phase the small energy barrier shrinks and is eventually overcome [Fig. 4(g)]. Thus the system leaves the metastable state, suddenly increasing the photon number in the cavity.

Termination and cyclic repetition of the pulses may be qualitatively understood from the dynamic dispersive shift that depends on the overlap between cavity mode and atomic wave function. The strong localization of the atoms at the nodes of the cavity mode during a spike can reduce the dispersive shift until the effective cavity resonance is crossed, terminating self-ordering. The cavity 
mode empties and the process repeats until heating of the BEC and atom loss finally stop the process. A theoretical description of the observed complex time dynamic phenomena requires a master equation approach, which goes beyond the scope of this work.

We demonstrated the existence of stable and dynamic self-ordered phases of a Bose-Einstein condensate coupled to a high-finesse optical cavity mode via a repulsive optical lattice. A theoretical description of the phase boundaries revealed that an antisymmetric coupling to the $P$ band of the pump lattice induces self-organization. This different lattice geometry for $\Delta_{a}>0$ could further lead to a qualitatively different coupling behavior in two- or multimode scenarios [17,32]. The observed cyclic repetition of pulses appears qualitatively in the phase diagram where limit cycles, chaos, and time crystal behavior were theoretically predicted in related models [26-28]. We presented a novel approach for experimentally and theoretically exploring time dynamics in driven-dissipative systems [33-39].

We thank Nishant Dogra, Manuele Landini, Francesco Piazza, and Helmut Ritsch for insightful discussions. We acknowledge funding from SNF: Projects No. 182650 and No. 175329 (NAQUAS QuantERA) and NCCR QSIT, from EU Horizon 2020: ERC advanced grant TransQ (Project No. 742579) and ITN grant ColOpt (Project No. 721465), from SBFI (QUIC, Contract No. 15.0019), from EPSRC Grants No. EP/P009565/1 and No. EP/ K030094/1, and by an Investigator award of the Simons Foundation.

*donner@phys.ethz.ch

[1] H. Ritsch, P. Domokos, F. Brennecke, and T. Esslinger, Rev. Mod. Phys. 85, 553 (2013).

[2] S. Gopalakrishnan, B. L. Lev, and P. M. Goldbart, Nat. Phys. 5, 845 (2009).

[3] M. Müller, P. Strack, and S. Sachdev, Phys. Rev. A 86, 023604 (2012).

[4] F. Piazza and P. Strack, Phys. Rev. Lett. 112, 143003 (2014).

[5] J. Keeling, M. J. Bhaseen, and B. D. Simons, Phys. Rev. Lett. 112, 143002 (2014).

[6] R. Chitra and O. Zilberberg, Phys. Rev. A 92, 023815 (2015).

[7] S. Schütz, S. B. Jäger, and G. Morigi, Phys. Rev. Lett. 117, 083001 (2016).

[8] L. Himbert, C. Cormick, R. Kraus, S. Sharma, and G. Morigi, Phys. Rev. A 99, 043633 (2019).

[9] P. Kirton, M. M. Roses, J. Keeling, and E. G. D. Torre, Adv. Quantum Technol. 2, 1800043 (2018).

[10] Y. Li, L. He, and W. Hofstetter, Phys. Rev. A 87, 051604(R) (2013).

[11] M. Soriente, T. Donner, R. Chitra, and O. Zilberberg, Phys. Rev. Lett. 120, 183603 (2018).
[12] K. Baumann, C. Guerlin, F. Brennecke, and T. Esslinger, Nature (London) 464, 1301 (2010).

[13] J. Klinder, H. Keßler, M. Wolke, L. Mathey, and A. Hemmerich, Proc. Natl. Acad. Sci. U.S.A. 112, 3290 (2015).

[14] J. Klinder, H. Keßler, M. R. Bakhtiari, M. Thorwart, and A. Hemmerich, Phys. Rev. Lett. 115, 230403 (2015).

[15] R. Landig, L. Hruby, N. Dogra, M. Landini, R. Mottl, T. Donner, and T. Esslinger, Nature (London) 532, 476 (2016).

[16] J. Léonard, A. Morales, P. Zupancic, T. Esslinger, and T. Donner, Nature (London) 543, 87 (2017).

[17] A. J. Kollár, A. T. Papageorge, V. D. Vaidya, Y. Guo, J. Keeling, and B. L. Lev, Nat. Commun. 8, 14386 (2017).

[18] L. Hruby, N. Dogra, M. Landini, T. Donner, and T. Esslinger, Proc. Natl. Acad. Sci. U.S.A. 115, 3279 (2018).

[19] M. Landini, N. Dogra, K. Kroeger, L. Hruby, T. Donner, and T. Esslinger, Phys. Rev. Lett. 120, 223602 (2018).

[20] R. M. Kroeze, Y. Guo, V. D. Vaidya, J. Keeling, and B. L. Lev, Phys. Rev. Lett. 121, 163601 (2018).

[21] V. D. Vaidya, Y. Guo, R. M. Kroeze, K. E. Ballantine, A. J. Kollár, J. Keeling, and B. L. Lev, Phys. Rev. X 8, 011002 (2018).

[22] J. Keeling, M. J. Bhaseen, and B. D. Simons, Phys. Rev. Lett. 105, 043001 (2010).

[23] N. Liu, J. Lian, J. Ma, L. Xiao, G. Chen, J. Q. Liang, and S. Jia, Phys. Rev. A 83, 033601 (2011).

[24] M. J. Bhaseen, J. Mayoh, B. D. Simons, and J. Keeling, Phys. Rev. A 85, 013817 (2012).

[25] F. Mivehvar, H. Ritsch, and F. Piazza, Phys. Rev. Lett. 118, 073602 (2017).

[26] M. Diver, G. R. M. Robb, and G.-L. Oppo, Phys. Rev. A 89, 033602 (2014).

[27] F. Piazza and H. Ritsch, Phys. Rev. Lett. 115, 163601 (2015).

[28] H. Keßler, J. G. Cosme, M. Hemmerling, L. Mathey, and A. Hemmerich, Phys. Rev. A 99, 053605 (2019).

[29] See Supplemental Material at http://link.aps.org/ supplemental/10.1103/PhysRevLett.123.233601 for experimental details and derivations.

[30] C. Maschler, I. B. Mekhov, and H. Ritsch, Eur. Phys. J. D 46, 545 (2008).

[31] R. Mottl, F. Brennecke, K. Baumann, R. Landig, T. Donner, and T. Esslinger, Science 336, 1570 (2012).

[32] A. Morales, P. Zupancic, J. Léonard, T. Esslinger, and T. Donner, Nat. Mater. 17, 686 (2018).

[33] N. Dogra, M. Landini, K. Kroeger, L. Hruby, T. Donner, and T. Esslinger, arXiv:1901.05974.

[34] E. I. Rodriguez Chiacchio and A. Nunnenkamp, Phys. Rev. Lett. 122, 193605 (2019).

[35] I. Carusotto and C. Ciuti, Rev. Mod. Phys. 85, 299 (2013).

[36] S. Diehl, A. Tomadin, A. Micheli, R. Fazio, and P. Zoller, Phys. Rev. Lett. 105, 015702 (2010).

[37] B. Buča, J. Tindall, and D. Jaksch, Nat. Commun. 10, 1730 (2019).

[38] J. Kohler, J. A. Gerber, E. Dowd, and D. M. Stamper-Kurn, Phys. Rev. Lett. 120, 013601 (2018).

[39] P. Molignini, L. Papariello, A. U. J. Lode, and R. Chitra, Phys. Rev. A 98, 053620 (2018). 